\title{
Survey on Chemical Pathology Teaching in Medical Curricula: Insight into Patient Safety
}

\author{
Nike Kwai Cheung LAU, Ka Ngai LAU and Chloe Miu MAK* \\ Chemical Pathology Laboratory, Department of Pathology, Princess Margaret Hospital, China
}

Received: February 02, 2018; Published: February 12, 2018

*Corresponding author: Chloe Miu MAK, Chemical Pathology Laboratory, Department of Pathology, Princess Margaret Hospital, Hong Kong SAR, China, Tel: 852-29901882; Fax: 852-23700969; Email: makm@ha.org.hk

\begin{abstract}
Context: Proper utilisation and interpretation of laboratory test results depend on the understanding of the indications and characteristics of such tests. Undergraduate teaching on this is essential to patient safety.

Methods: Medical students were invited to comment on Chemical Pathology teaching, and their knowledge on interpretation of iron profile was tested. We also interviewed practising doctors on their opinion in Chemical Pathology interpretation service.

Results: We identified topics which students recognised as being taught, understood, or requiring more teaching. They were dissatisfied with the teaching in Chemical Pathology, despite their perception of it being clinically useful. Only one-third of students correctly interpreted the iron profile. Majority agreed that increase in teaching time (58.6\%), teaching in the clinical years (64.3\%) and teaching by Chemical Pathologists (65.7\%) would improve the teaching. Most practising doctors would like to have interpretation service on endocrine tests (63\%), acute phase markers (7.4\%), and electrolytes (7.4\%).
\end{abstract}

Conclusion: Current teaching in Chemical Pathology could be further strengthened from the students' perspective with more emphasis on clinical applications with real-life examples and laboratory-based tutorials would better prepare medical graduates on laboratory medicine and safeguard patient care. Chemical Pathology interpretation services should be implemented to further improve clinical practice.

Keywords: Chemical pathology; Undergraduate medical education; Medical curriculum; Pathology teaching; Hong Kong

\section{Introduction}

Chemical pathology is a branch of laboratory medicine in which biochemical methods are applied to the study, screening, diagnosis, treatment, and monitoring of diseases. In particular, appropriate utilization of the laboratory investigations and proper interpretation of the results require a thorough knowledge in both the analytical techniques and the clinical-biochemical basis of the diseases. Chemical pathology is a well-recognized specialty. The discipline originated in the late 19th century with the use of simple chemical tests to detect various analytes in blood, urine and other bodily fluids. Subsequently, more sophisticated techniques are applied including the use and measurement of enzyme activities, immunoassays, spectrophotometry, electrophoresis, chromatography, mass spectrometry, and genetic analysis.

Chemical pathology is the area of pathology that is generally concerned with analysis of bodily fluids. All biochemical tests come under chemical pathology which can cover up to about 700 different kinds of tests and continue to grow rapidly. Millions of laboratory tests are performed every year and consume a significant amount of the health care budget. This large array of tests can be further sub- categorized into general chemistry, special proteins, endocrinology, metabolic medicine, therapeutic drug monitoring and toxicology, heavy metals, tumour markers, point-of-care testing, genetics and genomics, and, in some areas, immunology is also included. The main functions of chemical pathologists are to provide specialistled laboratory services for the investigation and management of biochemical disorders, to interpret these investigations and, increasingly, to provide clinical services for patients with metabolic disorders.

The delivery of a chemical pathology service has become more and more complex over recent years with the advent of technology-driven era in terms of both the underlying chemistries and its technologies. Similar to other technology-driven disciplines, the services offered by the chemical pathology laboratory are indispensable to the care of most medical conditions, both acute and chronic. The clinical and chemical pathology skills required to support such a service must be based on a good and comprehensive educational system. Undergraduate teaching of chemical pathology is of utmost importance to enable medical students aptly apply 
knowledge of normal and disturbed physiology and biochemistry to solve clinical problems, to interpret biochemical test results against the background of a broad knowledge of factors affecting results, and to manage a rational and effective use of biochemical investigations.

Patient safety is a global imperative defined by the World Health Organisation (WHO) as "the reduction of risk of unnecessary harm associated with healthcare to an acceptable minimum" [1]. Primum non nocere, meaning "first, do no harm", despite being a well-known axiom amongst medical practitioners since the 19th century, is still one of the fundamental principles of contemporary medical ethics [2]. Patient safety is a serious global health issue requiring joint effort from all around the world to minimise unnecessary patient harm. One of the greatest challenges in improving patient safety is diagnostic error, which is "the mistake or failure in the diagnostic process leading to a misdiagnosis, a missed diagnosis, or a delayed diagnosis" [3]. The incidence of diagnostic errors which would have implications on clinical management, was estimated to be about $10-20 \%$ in various studies such as autopsy findings and crosssectional studies and even up to $28.6 \%$ in a US study using data from malpractice claims $[4,5]$.

As a consequence, such adverse patient outcomes have a significant impact on the economic cost of healthcare. About 20 $40 \%$ of all healthcare spending, as estimated by the WHO, is directed towards additional hospitalisation, litigation, infections, disability, lost productivity and medical expenses due to issues in patient safety [1]. Apart from these direct costs, the indirect economic burden of "defensive medicine" with unnecessary diagnostic tests to safeguard the clinician against lawsuits also aggravates the current situation, taking another US\$ $45-60$ billion every year in the USA [6]. Diagnostic errors have a pivotal role in health economics. Up to $44 \%$ of diagnostic errors are related to the testing process [3]. In the context of laboratory testing, these errors can originate from one or more of the steps of the total testing process, which includes pre-pre-analytical (test requesting), pre-analytical (test implementing and specimen collection), analytical post-analytical (reporting of results) and post-post-analytical (misinterpretation of test results) processes $[7,8]$. Laboratory analytical errors make up from 7.3 to $15 \%$ of all errors in the total testing process [9]. This means more than $80 \%$ of diagnostic errors originate from outside the laboratory.

At the frontline, the interns and junior doctors most often have to request and interpret diagnostic test results for immediate management of their patients. The doctors' knowledge on these diagnostic tests would be crucial to the proper utilisation of such tests. Before they acquire experience from bedside practice, the education they receive as medical students would be the most important factor to determine whether they could offer the most appropriate test and act accordingly to the results when they become clinicians. In a UK study, a questionnaire distributed to doctors in Foundation year 1 and Foundation year 2 (equivalent to their first two years of clinical practice after graduation) showed that more than $30 \%$ of them lacked confidence in the interpretation of common biochemical tests such as serum proteins, parathyroid hormone, short Synacthen test, and urine sodium and osmolality [10]. Also, $50 \%$ of them were not confident in the interpretation of haemolysed samples. Another study in South Africa showed similar findings amongst interns [11].

As recognised by the Centres for Disease Control and Prevention in US, the lack of education in laboratory testing, compounded by the increasing types and complexity of new laboratory tests, would contribute to the overall inadequacy [12]. It is conceivable that more diagnostic errors could arise if future doctors are not properly educated on the basic principles and the correct interpretation of essential laboratory tests. We conducted a local survey on medical students' knowledge and competence of chemical pathology in order to determine the area of deficiency. Relevant publications were searched and reviewed to formulate plans for improving the current medical teaching on chemical pathology.

\section{Materials and Methods}

A first voluntary questionnaire consisting of 8 questions (supplementary data) was distributed randomly electronically to medical students of the two local medical schools (The University of Hong Kong and The Chinese University of Hong Kong) who were in their clinical years of study. Anonymity of their identities was ensured. Questions were set to evaluate the existing scenario of teaching on Chemical Pathology: whether they have received proper teaching on Chemical Pathology, and in which year they received any teaching in Chemical Pathology. Then students were invited to self-evaluate their understanding on several common topics in Chemical Pathology, as well as how satisfied they were with their current curriculum of Chemical Pathology. Their perceptions of usefulness of chemical pathology in their future medical practice were also graded. They were invited to provide suggestions on how undergraduate chemical pathology teaching could be improved as well. The students were asked to interpret an iron profile as part of a clinical case scenario (adapted from the author's publication) [13].

The case scenario was about an elderly admitted for fever with a past history of congestive heart disease and primary hypothyroidism on thyroxine replacement. She was found to be anaemic with the presence of anisocytosis, microcytosis and hypochromasia. Iron profile showed a low serum iron, low total iron binding capacity, low iron saturation percentage and high ferritin. It was a picture of anaemia of chronic disease instead of iron deficiency. Students were then asked for any further investigations or treatment they would consider such as faecal occult blood testing, endoscopy, and iron supplement. A second survey consisting of an open question was also distributed to practising doctors in the specialties of Internal Medicine and Family Medicine for their opinions in what biochemical tests they would have difficulties in interpretation and would like to have input from Chemical Pathologists.

\section{Results}

\section{Test coverage}

For the first survey, a total of 70 responses were received from the medical students. Over $98 \%$ of the medical students stated that 
they have received teaching on Chemical Pathology throughout their medical education.

\section{Some Tests are Better Taught and Understood}

Most of the common topics in Chemical Pathology were perceived to be taught by over $50 \%$ of the students (Figure 1). Topics which were perceived as taught by over $75 \%$ of the students include "lipid and lipoproteins", "sodium and water metabolism", "acid-base and blood gas", "calcium, phosphate and magnesium" and "potassium metabolism". Topics self-evaluated to be best understood include "thyroid gland", "sodium and water metabolism" and "acid-base and blood gas" (Figure 2).

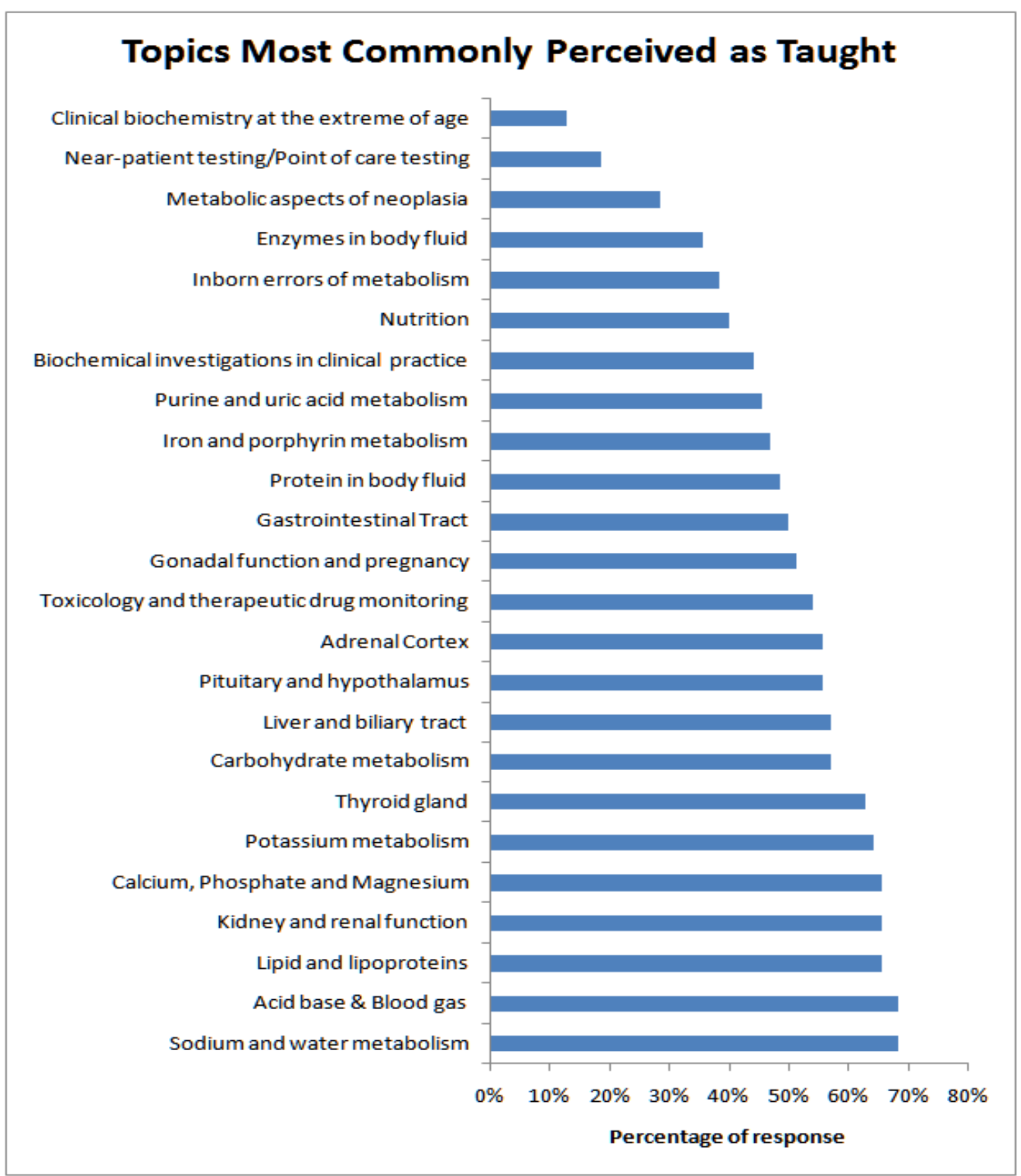

Figure 1: Percentage of response from medical students on the topics most commonly perceived as taught in medical school.

\section{Some topics are not well taught and poorly understood}

Notably, topics such as "metabolic aspects of neoplasia", "pointof-care testing" and "clinical biochemistry at the extremes of age" were only perceived by less than half of the students to be taught. Similarly, topics such as "point-of-care testing", "inborn errors of metabolism" and "clinical biochemistry at the extreme of age" were amongst the most poorly understood ones (Figure 2). 


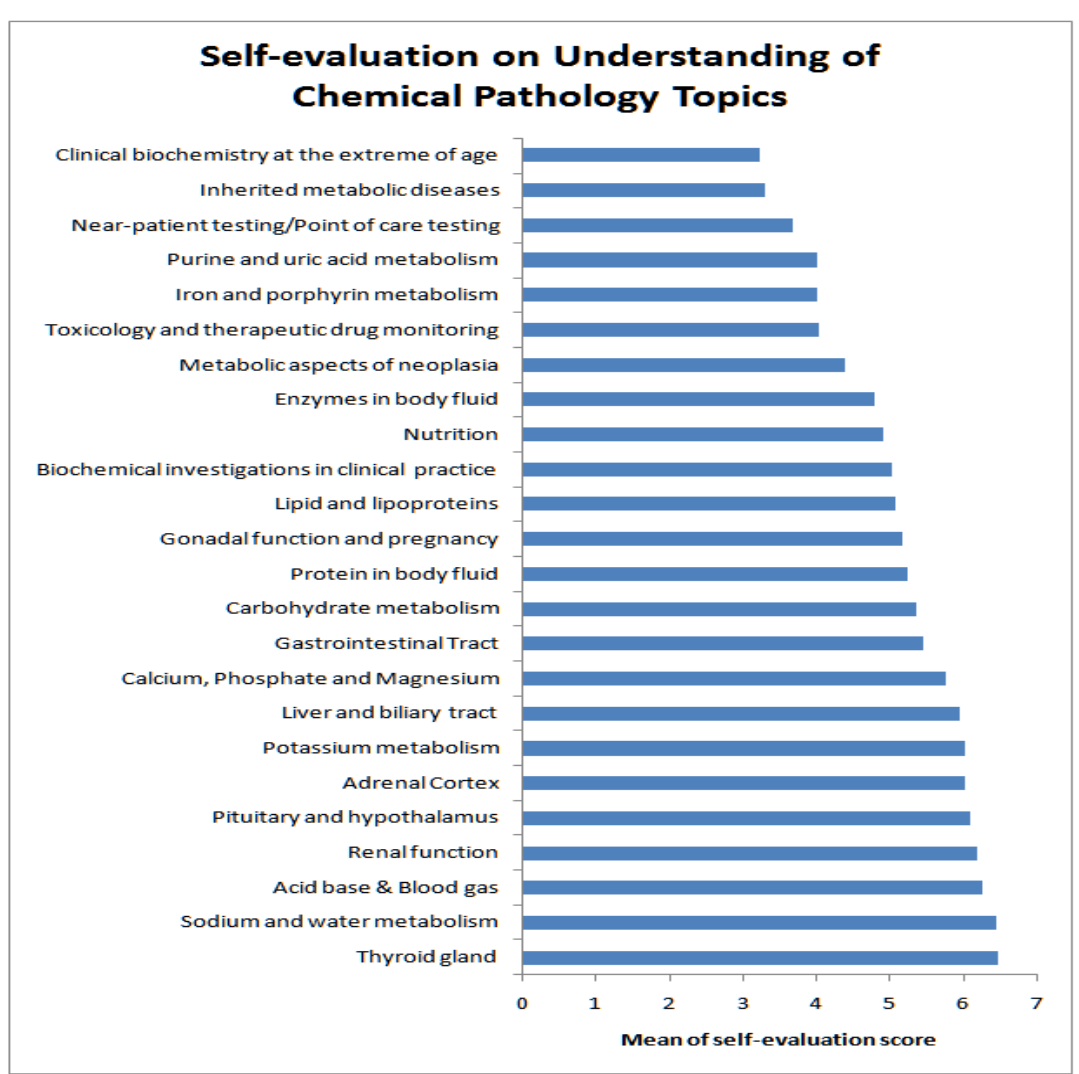

Figure 2: Mean score of response from medical students on self-evaluation on the understanding of each topic.

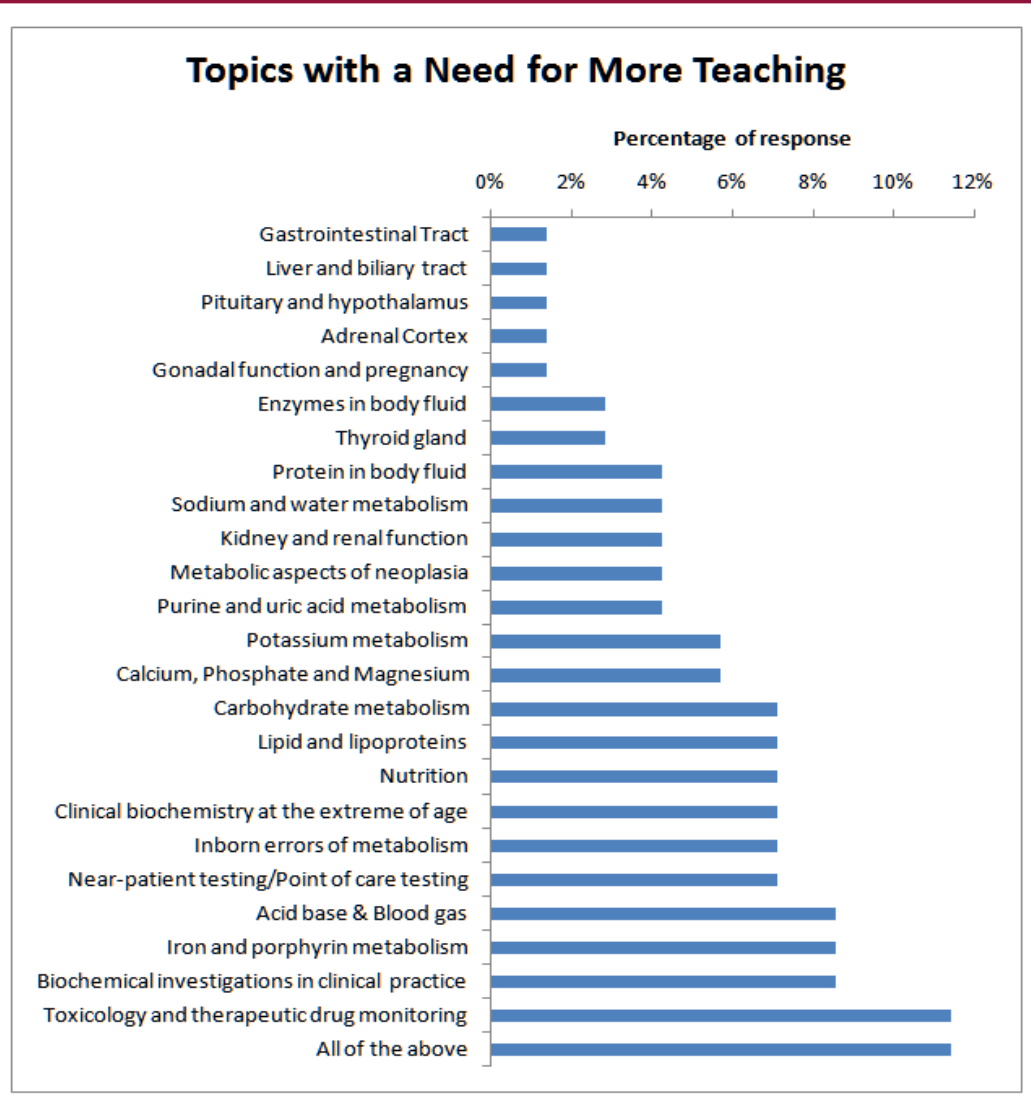

Figure 3: Percentage of response from medical students on the topics with a need for more teaching. 


\section{Topics students perceived as needed}

Interestingly, when asked which topic should be taught more, "toxicology and therapeutic drug are monitoring", "acid base \& blood gas", "iron and porphyrin metabolism" and "biochemical investigations in clinical practice" were the most popular topics. However, "all of the topics" were the most chosen option when asked what should be taught more (Figure 3).
Chemical Pathology is perceived as important but teaching is not satisfactory

The mean satisfaction score (on a scale of 0 [not satisfied] to 10 [very satisfied]) was 5.4, which contrasted with the mean score of 7.9 (on a scale of 0 [not useful] to 10 [very useful]) for the perceived usefulness of Chemical Pathology in their future medical practice (Figure 4 \&5).

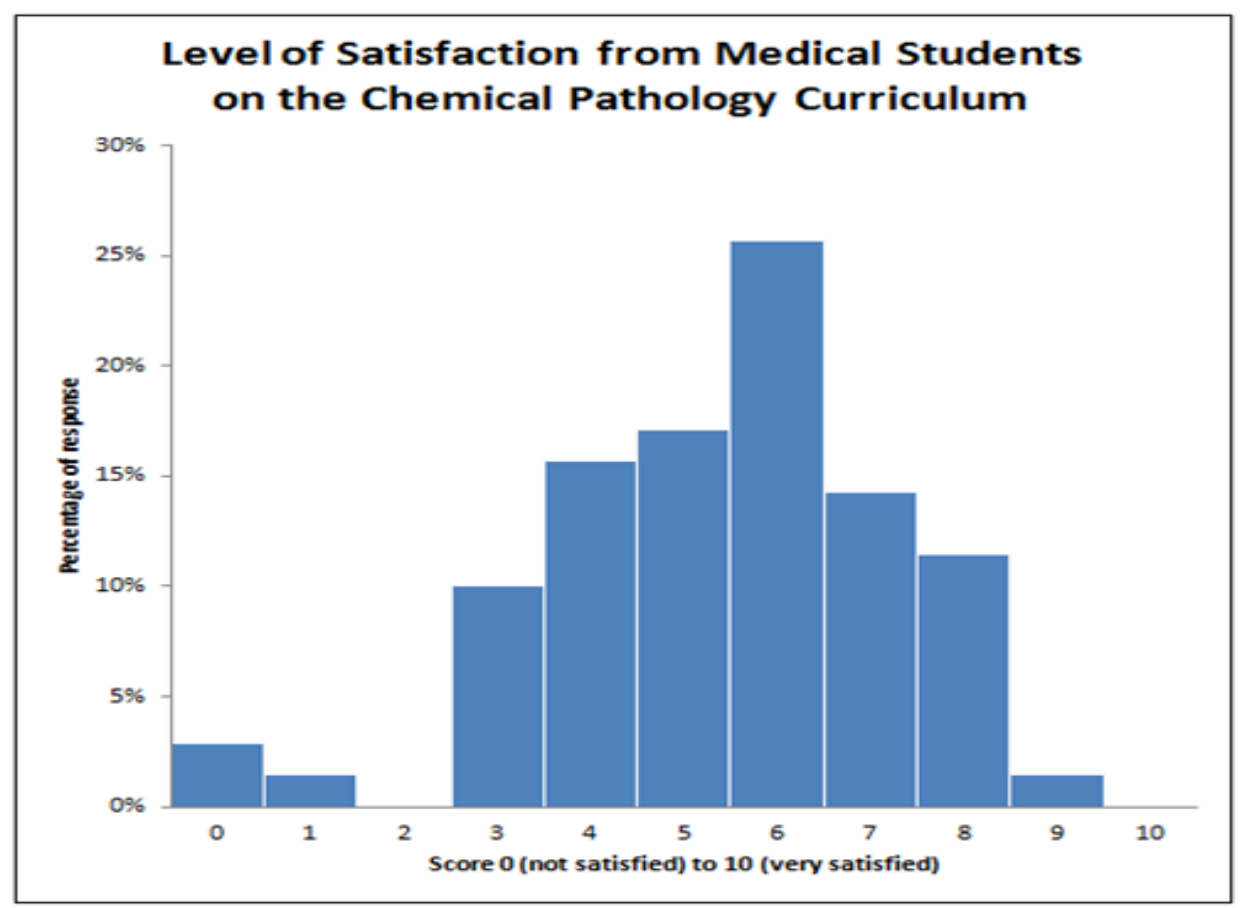

Figure 4: Percentage of response from medical students on the satisfaction of students on current curriculum.

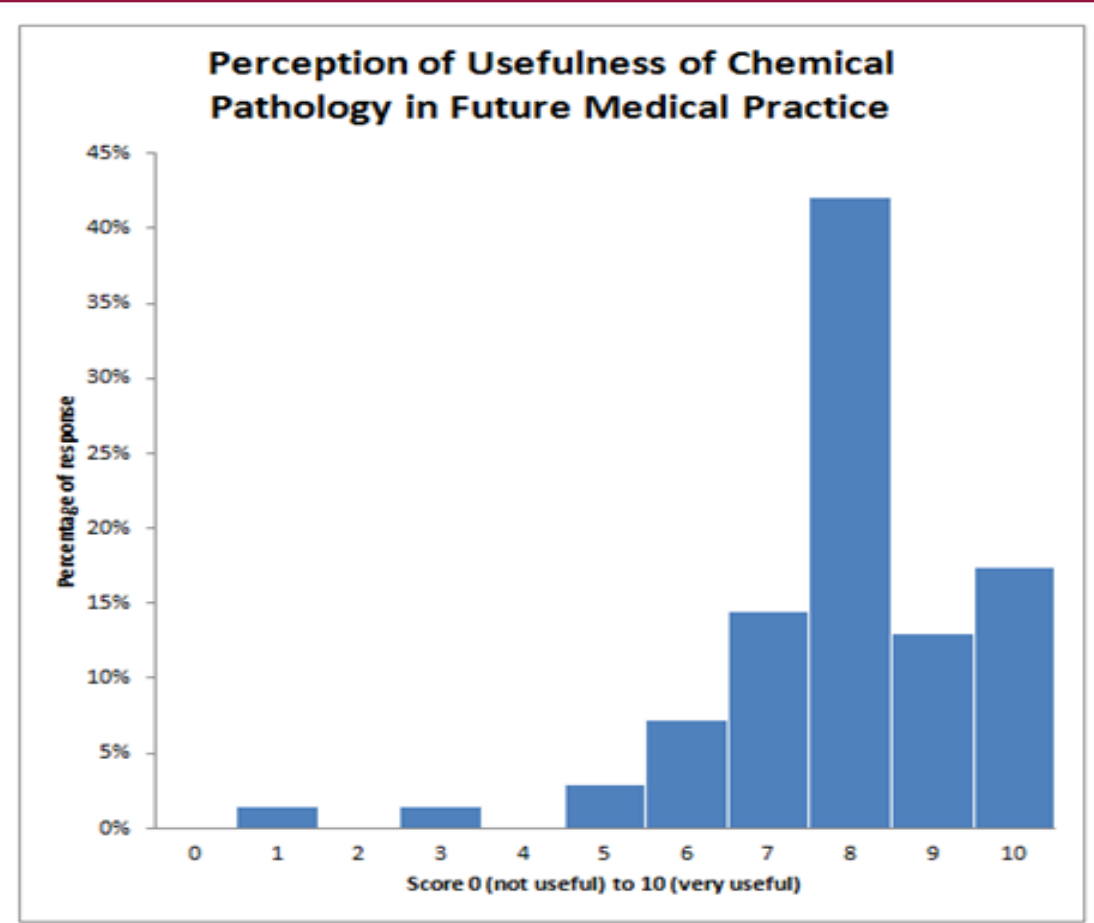

Figure 5: Percentage of response from medical students on the perception of usefulness of Chemical Pathology in future medical practice. 


\section{Suggestions to improve teaching}

The possible ways to improve the current curriculum as suggested by the students were categorised and include more emphasis on the clinical applications of the topics, more lectures, exercise or problem-based learning sessions. The majority of students agreed that increase in teaching time (58.6\%), teaching in the clinical years (64.3\%) and teaching by Chemical Pathologists (65.7\%) would help improving the curriculum. Other responses include project-based teaching; focus on quality of teaching more than quantity, laboratory-based tutorials on analytical principles, as well as examples of laboratory reports on common clinical problems (Figure 6).

\section{Most students Cannot Interpret the Iron Profile correctly}

The iron profile consisted of two questions, assessing if the students understand the basic pre-analytical requirements of the iron profile testing and post-analytical interpretation of a clinical scenario. Only $31.3 \%$ and $32.3 \%$ of students were able to provide a correct answer for the two questions respectively. For students who were uncertain about the diagnosis, most would suggest repeating a fasting morning iron profile when metabolically stable. For students who interpreted the result as iron deficiency (32.5\%), four out of twenty (20\%) of them suggested further investigations for anaemia including faecal occult blood testing and endoscopy. A portion of students also suggested a therapeutic trial of iron supplement for the patient.

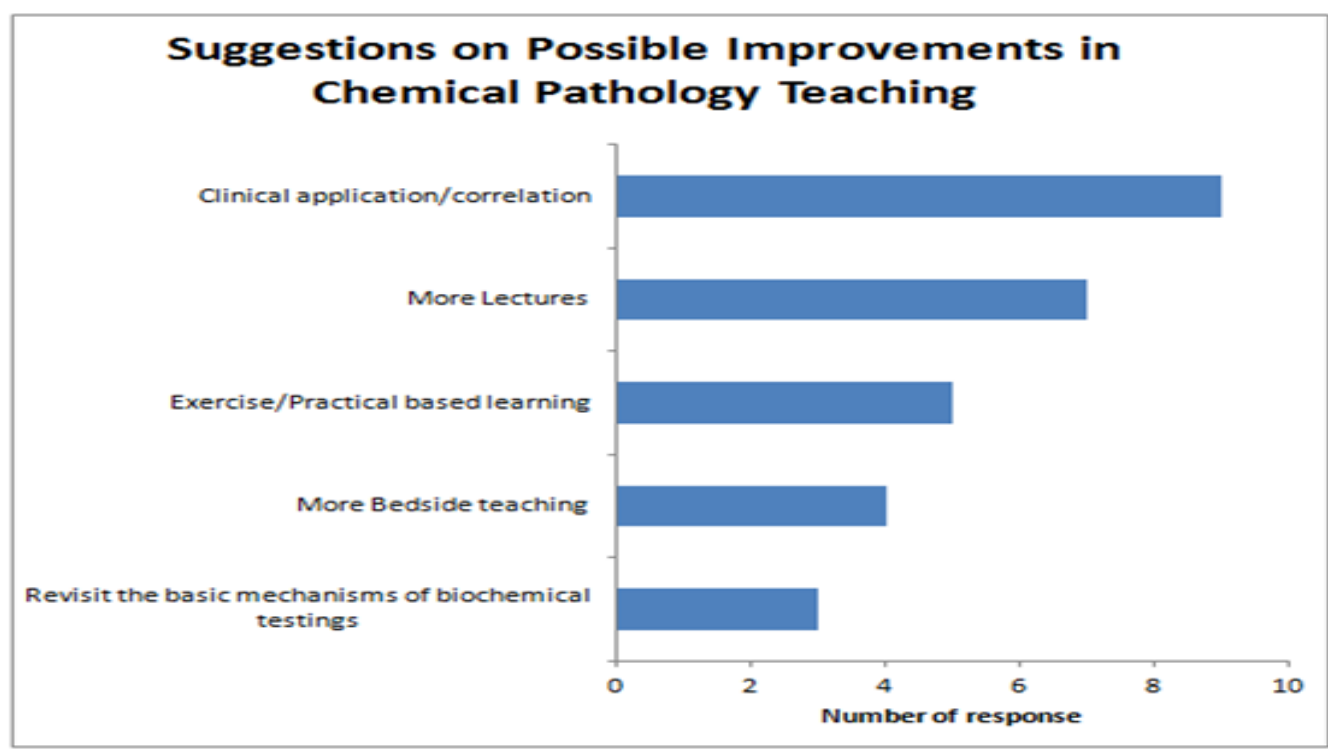

Figure 6: Suggestions and the number of response from medical students on possible improvements in Chemical Pathology teaching.

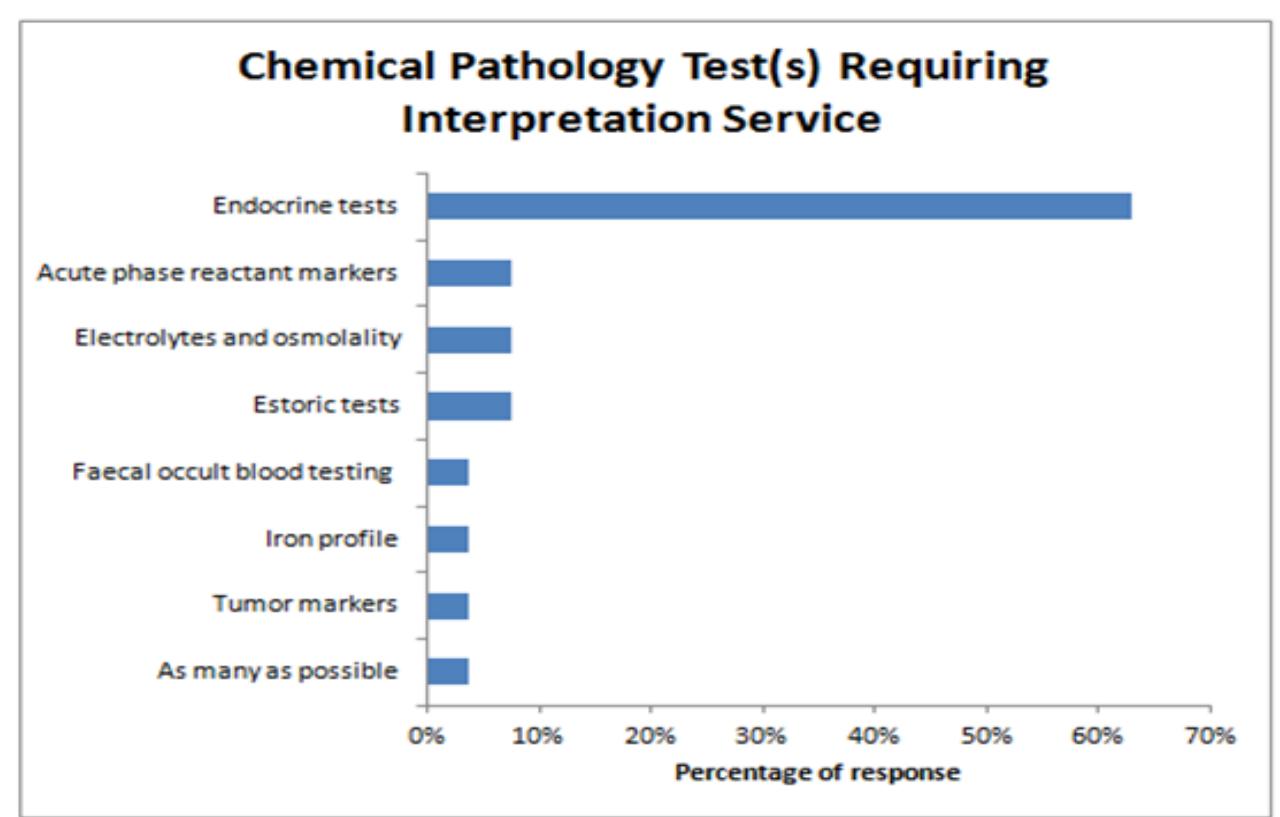

Figure 7: Percentage of response from clinicians on which Chemical Pathology test(s) they would prefer interpretation service. 


\section{Doctors Want Help in Endocrine Tests}

For the second survey, we received 12 responses from Internal Medicine doctors and 11 responses from doctors in Family Medicine. Most doctors would like to receive Chemical Pathology interpretation service on endocrine tests (63\%), acute phase reactant markers $(7.4 \%)$, electrolytes and osmolality $(7.4 \%)$ and rare tests such as fluid amylase (7.4\%). Amongst endocrine tests, results on serum cortisol, renin, aldosterone and their dynamic function tests such as short synacthen test and postural test are perceived as the most difficult ones. Internal medicine physicians request mainly for interpretation on endocrine tests while family physicians prefer also on electrolytes and tumour markers apart from endocrine tests (Figure 7).

\section{Discussion}

Medical students agreed that Chemical Pathology is an important subject for their future clinical practice but with unsatisfactory teaching in the undergraduate medical curriculum. In addition to the existing studies in the literature which suggested junior doctors as the important guardian of patient safety to prevent diagnostic errors[10,11], our study suggested another link between medical education and patient safety - medical students. Our results from the two surveys have demonstrated that there were knowledge deficiencies amongst medical students in requesting and interpreting biochemical tests, which may result in diagnostic errors. From the authors' experience, and also suggested in a previous study [11], it is usually difficult and not a common practice amongst public hospitals in Hong Kong to offer teaching for junior doctors on Chemical Pathology due to their hectic clinical duties. It is imperative to empower medical undergraduates with knowledge in laboratory medicine.

Some of the common biochemical tests which are encountered in routine medical practice, for example liver and renal function tests, endocrinology tests like thyroid function tests, pituitary and hypothalamic hormones as well as adrenal function tests, and the tests which may have potential life-threatening consequences, such as blood gas and electrolytes, are generally perceived as well taught and self-evaluated to be better understood. Considerable deficits are observed in some specialised areas such as Oncology, Geriatrics, Paediatrics, as well as areas which require comprehensive knowledge in biochemical pathways such as inborn errors of metabolism. Given these specialties occupied a relatively smaller proportion of the whole medical curriculum; it may seem inevitable that the biochemical aspects of patients in these specialties may be less familiar to medical students. However, it is not without potential danger as exemplified by one of these topics, inborn errors of metabolism, that inability to request and interpret relevant test results could be potentially life-threatening. Proper knowledge in recognising the clinical presentation, requesting the appropriate diagnostic tests and making the correct interpretation and hence management would still be of paramount importance for medical students as they would be the frontline doctors managing patients in these specialties.
The test on iron profile interpretation for medical students served as an objective assessment of their ability to tackle common problems in Chemical Pathology. The deficiency in the teaching in Chemical Pathology could also be exemplified by the worrying results of the iron profile interpretation. Only one-third of the students could give a correct response as to how should the patient be prepared for the test (pre-analytical stage of testing) as well as whether the patient in the question was iron-deficient or not (post-analytical interpretation). The iron profile is one of the most commonly requested biochemical tests in clinical practice across many specialties including Internal Medicine, Surgery, Paediatrics and Obstetrics and Gynaecology as part of the investigation panel for anaemic patients. A fasting morning sample is recommended because the serum iron level varies diurnally and changes with dietary intake [13]. Incorrect interpretation of the iron profile may lead to unnecessary investigations such as faecal occult blood testing and endoscopic examinations, which are not without risks. Corresponding to the self-evaluation results, iron profile test is also graded amongst the worst understood topics and topics requiring more teaching.

To reflect on our existing curriculum, the current standards in the literature and the comments from our students should both be taken into consideration. From our study, the low level of satisfaction towards the current Chemical Pathology teaching could stem from the inadequate teaching hours, lack of clinical correlation or scenario based teaching, and lack of teaching from specialists in Chemical Pathology, as reflected from their recommendations on how the chemical pathology curriculum should be improved. In the literature, the features of overall success of laboratory medicine teaching have been described to include the followings: small core group of dedicated expert in laboratory medicine, small group discussion with close relationship between students and instructor, as well as case discussions focusing on clinical problem solving. Both the results from our students and the literature suggested the need of teaching of clinical cases by Chemical Pathologists. The limited resources such as number of Chemical Pathologists and course hours available would be less flexible for negotiation, but certainly the content and method of delivery of the contents have a room for improvements.

Teaching chemical pathology in clinical years is another frequent response to how to improve the current curriculum. Traditionally, Pathology is part of the pre-clinical curriculum which focuses more on basic biomedical scientific principles. As a result, the lack of clinical context may render the teaching a mere discussion of theoretical scenarios, and there may be difficulties to transfer the interpretation skills to clinical application on real patients. A review on how medical schools integrate pathology teaching into the clinical years postulated only $40 \%$ of medical schools in the United States and Canada provided exposure to pathology in the clinical years, ranging from around an hour to four weeks long [14]. Most of the required programs were integrated into the clinical clerkship and some of them even offered week-long independent module/clerkship with laboratory tour and clinical case based learning, covering topics like iron deficiency and gammopathy. 
One of the most frequent responses on potential areas for improvements is that teaching on all of the topics should be increased, which reflect a general feeling of inadequacy in Chemical Pathology teaching, given that they have graded Chemical Pathology as a useful subject in their future medical practice. Other topics the students wished for more teaching include toxicology and therapeutic drug monitoring, blood gas, iron and porphyrin metabolism and general biochemical investigations. Interestingly, these are not the topics which they self-evaluated to be the least understood ones. There may be a difference between what they thought to be important to know, maybe for examination purposes, and their actual knowledge deficiencies.

Alternative methods other than lecturing are also suggested, such as interactive problem-based teaching as well as laboratorybased hands-on practical sessions. Medical students now are 'digital natives', electronic learning support would be a useful supplement to the traditional lecturing. The benefits of mobile technology provision were demonstrated by a UK group, in which trainee doctors who were equipped with library texts on a smartphone reported improvement in provision of patient care in terms of precision in management planning [15]. With almost every student possessing a smartphone, a mobile application comprised of clinical scenarios and multiple-choice questions with feedback on responses can be developed to reinforce their knowledge learnt during lectures. Reliable information on appropriate laboratory test ordering, proper interpretation of the test results and followup management can be rendered readily assessable to students if also included in the same application.

On the other hand, practising doctors in Internal Medicine and Family Medicine, who have had experience in frontline clinical medicine, suggested biochemical tests in endocrinology are the most challenging ones and in particular thyroid and adrenal function tests. This reflects the difference between what medical students think they should know (mainly for examination purposes) and what clinicians think they wished to know more (mainly for challenging cases they encountered during their practice). Also, it demonstrated that even clinicians would notice challenges in their practice with regard to Chemical Pathology. There may be an unmet demand of Chemical Pathology consultation service for clinicians, which would potentially facilitate discussion on difficult clinical cases, for example in the area of endocrinology, to allow better informed clinical decisions to be made. In the authors' laboratory, we have consultation service available for clinicians to discuss with Chemical Pathologists should they encounter complicated biochemistry in their patients [16].

The two surveys cover both medical students and doctors and aim to highlight the contrast between medical education and real-life practice. However, these simple surveys are mainly selfevaluation based, which is mainly on the subjective perception of the respondent. As these surveys are electronically distributed, no information on the actual response rate is recorded and the number of responds we received is indeed suggestive, the sample size may not be representative of the whole population of clinical students. Although iron profile interpretation is an objective assessment of the ability of the students, it would be better if data from written examinations is used as it would reflect both the students' performance in the prepared state as well as a more representative population.

\section{Conclusion}

"To err is human", but when it comes to clinical decisions, mistakes can jeopardise patient safety, incur financial burden or even cost lives. With the example of Chemical Pathology, it should be recognised that the current undergraduate education may be inadequate and potential diagnostic errors could be made due to lack of sufficient knowledge. These adverse consequences are avoidable and amenable. We would suggest a review of the current undergraduate medical curriculum and further study into how to improve the teaching to safeguard patient safety. With the advancement of laboratory technologies and introduction of new tests, the role of Chemical Pathologists in teaching the future generation of doctors and supporting clinical decisions should be emphasized.

\section{References}

1. World Alliance For Patient Safety Drafting G, Sherman H, Castro G (2008) Towards an international classification for patient safety: the conceptual framework. Int J Qual Health Care 21(1): 2-8.

2. Smith CM (2005) Origin and uses of primum non nocere--above all, do no harm!. J Clin Pharmacol 45(4): 371-377.

3. Schiff GD, Hasan O, Kim S (2009) Diagnostic error in medicine: analysis of 583 physician-reported errors. Arch Intern Med 169(20): 1881-1887.

4. Graber ML (2013) The incidence of diagnostic error in medicine. BMJ Qual Saf 22(2): ii21-ii27.

5. Saber Tehrani AS, Lee H, Mathews SC (2013) 25-Year summary of US malpractice claims for diagnostic errors 1986-2010: an analysis from the National Practitioner Data Bank. BMJ Qual Saf 22: 672-680.

6. Newman-Toker DE, McDonald KM, Meltzer DO (2013) How much diagnostic safety can we afford, and how should we decide? A health economics perspective. BMJ Qual Saf 22(2): ii11-ii20.

7. Smith BR, Aguero-Rosenfeld M, Anastasi J (2010) Educating medical students in laboratory medicine: a proposed curriculum. Am J Clin Pathol 133(4): 533-542.

8. Laposata M, Dighe A (2007) "Pre-pre" and "post-post" analytical error: high-incidence patient safety hazards involving the clinical laboratory. Clin Chem Lab Med 45(6): 712-719.

9. Plebani M (2009) Exploring the iceberg of errors in laboratory medicine. Clin Chim Acta 404(1): 16-23.

10. Khromova V, Gray TA (2008) Learning needs in clinical biochemistry for doctors in foundation years. Ann Clin Biochem 45: 33-38.

11. Stanfliet JC, Macauley J, Pillay TS (2009) Quality of teaching in chemical pathology: ability of interns to order and interpret laboratory tests. J Clin Pathol 62(7): 664-666.

12. Wolcott J, Schwartz A, Goodman C (2008) Laboratory medicine: a national status report.

13. Mak C, Chiu R, Lam CW (2006) Chemical pathology case conference assessment of iron status in general practice. Hong Kong Pract 28(9): 376-382. 
14. Magid MS, Cambor CL (2012) The integration of pathology into the clinical years of undergraduate medical education: a survey and review of the literature. Hum Pathol 43(4): 567-576.

15. Hardyman W, Bullock A, Brown A (2013) Mobile technology supporting trainee doctors' workplace learning and patient care: an evaluation. BMC Med Educ 13: 6.

\section{(c) (i) \\ This work is licensed under Creative Commons Attribution 4.0 License}

Submission Link: http://biomedres.us/submit-manuscript.php
16. ak CM, Siu WK, Law CY (2012) Electronic chemical pathology consultation service and dried blood spot metabolic screening in hospital patients. J Clin Pathol 65(12):1141-1145.

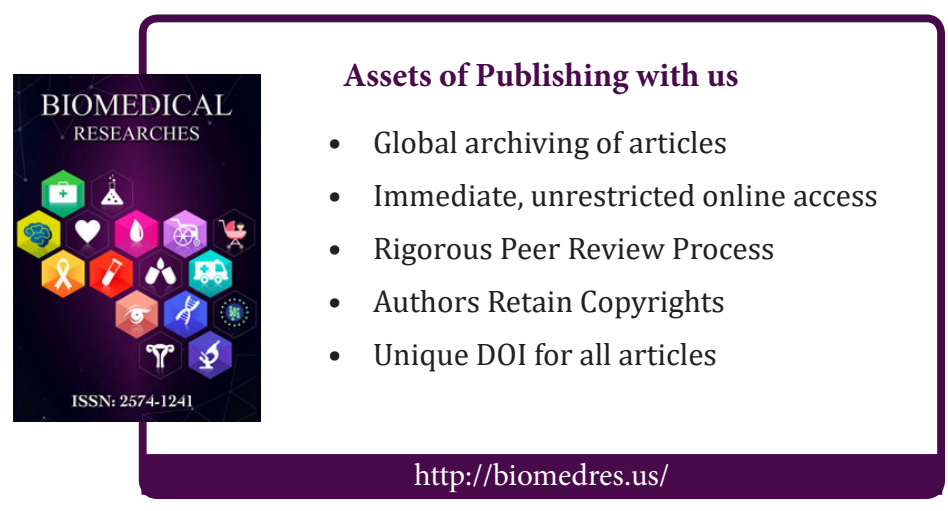

